\title{
Los Valerii de Ruscino: la rápida promoción de una familia de notables local
}

\section{The Valerii of Ruscino: the rapid promotion of a local family of notables}

\section{Marc Mayer i Olivé}

Institut d'Estudis Catalans / Universitat de Barcelona

Departament de Filologia Llatina

Gran Via de les Corts Catalanes, 585, E-08007 Barcelona

mayerolive@yahoo.es

The inscriptions that mention or seem to be ascribed to the Valerii of Ruscino present a homogeneity of forms that make their belonging to the same setting very credible. The reconstruction of their texts allows to examine the promotion of this family of local notables in a short period of time.

\section{KEY WORDS}

RUSCINO, ROMAN EPIGRAPHY, ROMAN SOCIETY, ROMAN HISTORY, GALLIA NARBONENSIS

Las inscripciones que mencionan o parecen poderse referir a los Valerii de Ruscino presentan una homogeneidad de formas que hacen muy verosímil su pertenencia a un mismo núcleo. La reconstrucción de sus textos permite ver la promoción en un corto espacio de tiempo de esta familia de notables locales. 
Un conjunto de cuatro inscripciones halladas en Ruscino, Château-Roussillon, Perpiñán (Pirineos Orientales) se refieren a la familia de los Valerii de esta ciudad. ${ }^{1}$ Las inscripciones en cuestión, si se acepta nuestra propuesta de que pertenezcan a un mismo núcleo, nos revelan a los Valerii como una de las familias más notables de una ciudad para la que los datos epigráficos referidos a su sociedad y organización son pocos, a pesar de los abundantes fragmentos de epígrafes dedicados a la domus julioclaudia. La nueva edición que preparamos de las inscripciones de la ciudad nos ha permitido nuevamente examinar estas inscripciones fragmentarias y proponer nuevas integraciones, así como una nueva agrupación de fragmentos en alguna ocasión, que permiten entender mucho mejor la entidad del conjunto que estudiamos, como indicaremos en cada caso.

Las inscripciones parecen darnos, como veremos a continuación, noticia de dos generaciones sucesivas de esta familia en época julioclaudia, un límite que no tiene nada de sorprendente, habida cuenta de la posterior destrucción de la ciudad al término de este período, que parece por el momento revelarnos la arqueología. Las inscripciones como consecuencia de esta destrucción se presentan de manera muy fragmentaria y los textos que proponemos responden a los resultados de una cuidadosa autopsia y de la reconstrucción que parece derivarse.

Señalemos en primer lugar que tres de ellas, ILGN 636, ILGN 632 y la constituida por un conjunto de fragmentos que contienen una parte de ILGN 632 en la integración realizada anteriormente por E. Esperandieu [n. ${ }^{\circ}$ inv. Rus.1914.00.3101; Rus.1914.00.3122 y Rus.1914.00.3124; Rus.1914.00.3123 y Rus.1914.00.3108, respectivamente], están grabadas en placas de mármol de Luni, Carrara, marmor Lunense, "bardiglio» y tienen la particularidad de ser muy delgadas, ya que su espesor varía entre 0,8 y $2,3 \mathrm{~cm}$. Sus dimensiones reconstruibles se mueven en torno a los $90 \mathrm{~cm}$ de altura y a los 60 de anchura. Una cuarta inscripción de este mismo material, ILGN 633, es la que los ciudadanos de Ruscino erigieron a Publius Memmius Regulus, el cónsul suffectus del 31 dC, y contribuye en buena medida, por su cronología interna muy aproximada, a datar las otras tres del mismo material y características, dado que dicho personaje es honrado posiblemente al término de su carrera como patronus de la ciudad. ${ }^{2}$

Las otras dos inscripciones que se refieren explícitamente a los Valerii no corresponden a esta misma tipología, ya que están grabadas en sendas placas relativamente gruesas de marmor Docimium, "pavonazzetto" de Asia Menor. En la primera, es honrado un C. Valerius Paetus, ILGN 635 = AE 1980, 615 [nº. inv. Rus.1914.00.3107 y Rus.1914.00.3106] (fig. 1) de quien se indica que fue el primero entre los de Ruscino en alcanzar el ordo equester y en haber obtenido todos los cargos ciudadanos; la segunda está dedicada a un L. Valerius y presenta la particularidad de ser opistógrafa [n. ${ }^{\circ}$ inv. Rus.1914.00.3103] (fig. 2) ${ }^{3}$ y lle-

1. Este trabajo se inscribe en el proyecto FFI2015-68571, en el seno del Grup consolidat LITTERA 2017 SGR241 de la Universitat de Barcelona y del Programa d'Epigrafia Llatina del Institut d'Estudis Catalans.

2. Número de inventario en el Centre Rémy Marichal de Ruscino, Rus.1914.00.3099; ILGN 633. Sobre esta inscripción cf. Mayer (e.p.), con la bibliografía anterior.

3. No recogida en ILGN, pero cf. Gayraud ,1980: 76, n. ${ }^{\circ} 5$. 


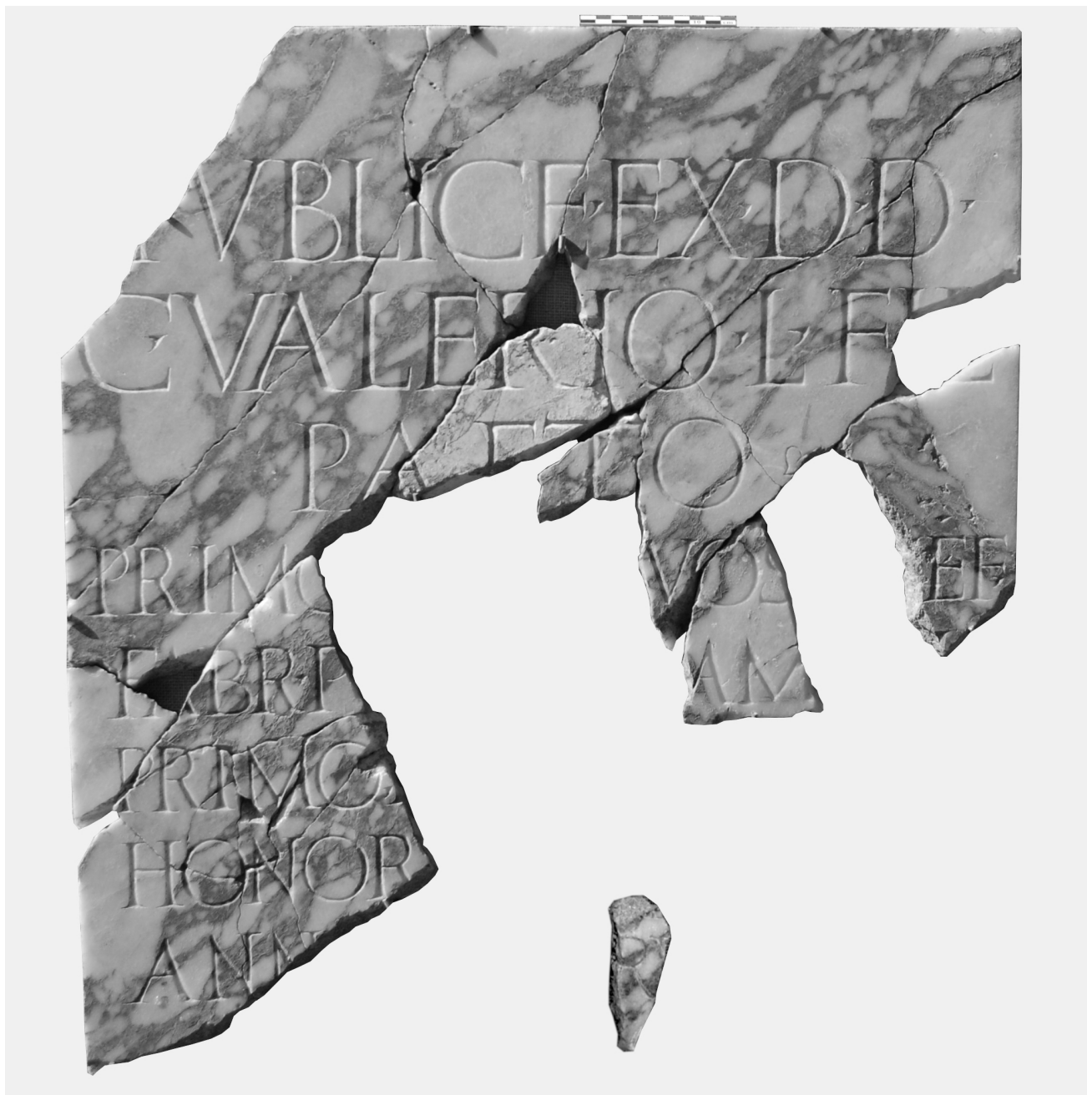

Figura 1. ILGN $635=A E 1980,615$ [n. ${ }^{\circ}$ inv. Rus. 1914.00 .3107 y Rus. 1914.00 .3106$]$.

var grabada en la cara opuesta una dedicatoria a Calígula, ILGN 620 (fig. 3), que parece evidentemente anterior, ya que su texto está recortado para su reutilización, por lo cual debemos datarla como mínimo en el reinado de Claudio, cosa que conllevaría una cronología semejante para la otra inscripción del mismo material y tipo.

Tendríamos así al menos dos generaciones de Valerii que vendrían a ilustrar su promoción social mediante la erección consiguiente de estatuas honoríficas. La primera respondería seguramente a las dos inscripciones en marmor Docimium y, la segunda, se serviría de las inscripciones de «bardiglio». 


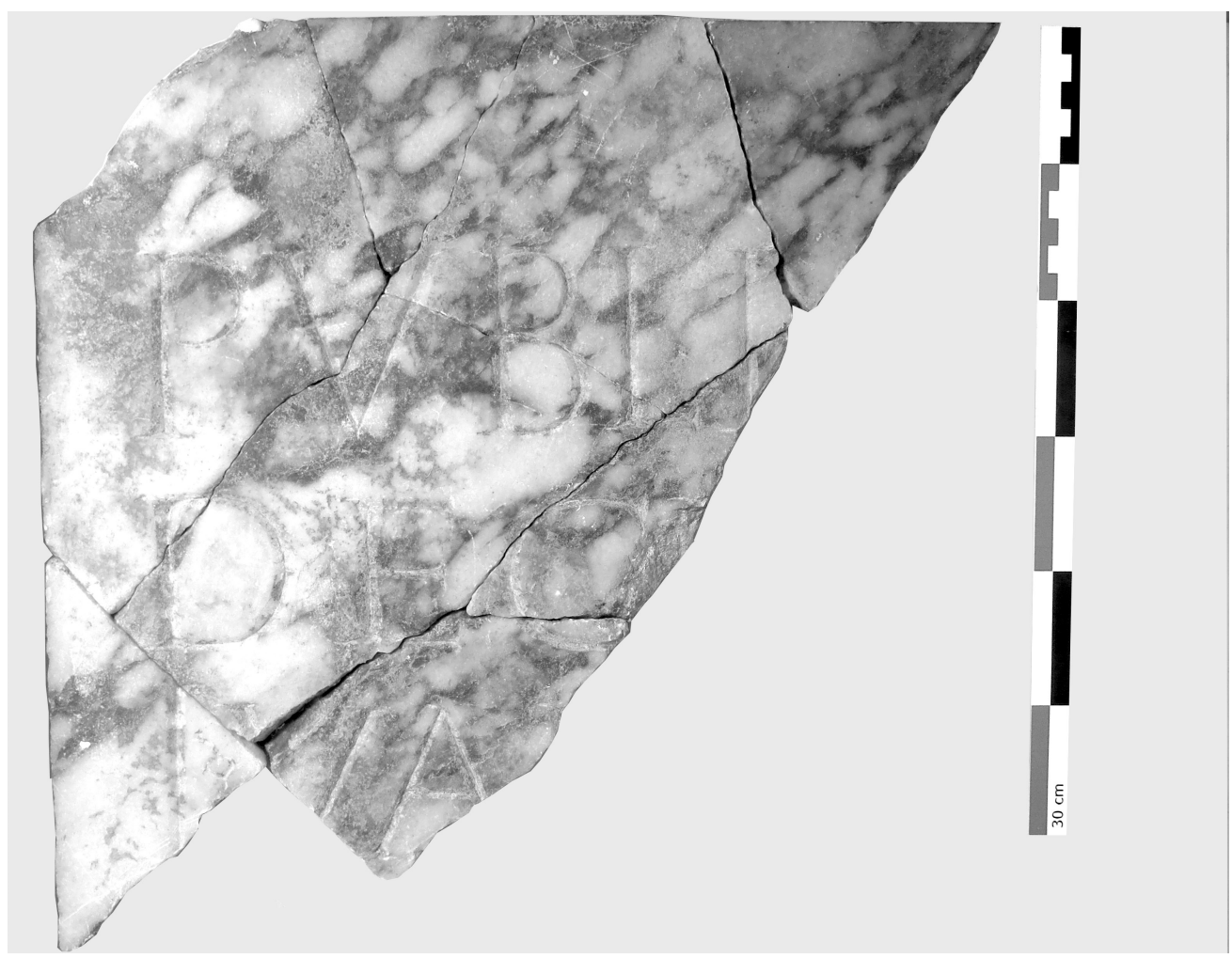

Figura 2. Gayraud 5 b [n. ${ }^{\circ}$ inv. Rus.1914.00.3103].

Para honrar esta segunda generación se constituiría un conjunto de cuatro inscripciones de «bardiglio» de Carrara muy semejantes entre sí, en el que, además de la inscripción de P. Memmius Regulus, ILGN 633 (fig. 4) a la que ya nos hemos referido, estaría presente la de C. Valerius C. f. Maxumus, ILGN 636 (fig. 5), un legado de rango senatorial; otra placa estaría dedicada a un Maxumus, ILGN 632 (fig. 6), seguramente otro Valerius, que alcanzó el grado de tribuno militar, y cuyo epígrafe presenta las mismas características que la anterior, a lo que se sumaría la posible indicación del patronato, y una cuarta está dedicada a un personaje anónimo que alcanzó el rango ecuestre por concesión de Claudio y que había sido praefectus y praepositus de la classis praetoria de Miseno (fig. 7), un fragmento de la cual Esperandieu, ILGN 632, en su momento, había propuesto añadir a la inscripción ya mencionada con este número (fig. 5), ${ }^{4}$ aunque se trata como veremos de una inscripción independiente de las reconstrucciones hasta ahora propuestas e integrable de manera distinta. El parecido con las dos anteriores quizá nos llevaría también a pensar en

4. Mayer, 2016a: 142-143, con fotografía de los fragmentos. Sobre algunos elementos de este conjunto puede verse una primera aproximación parcial en Mayer, 2016b: 19-44, esp. 31-36. 


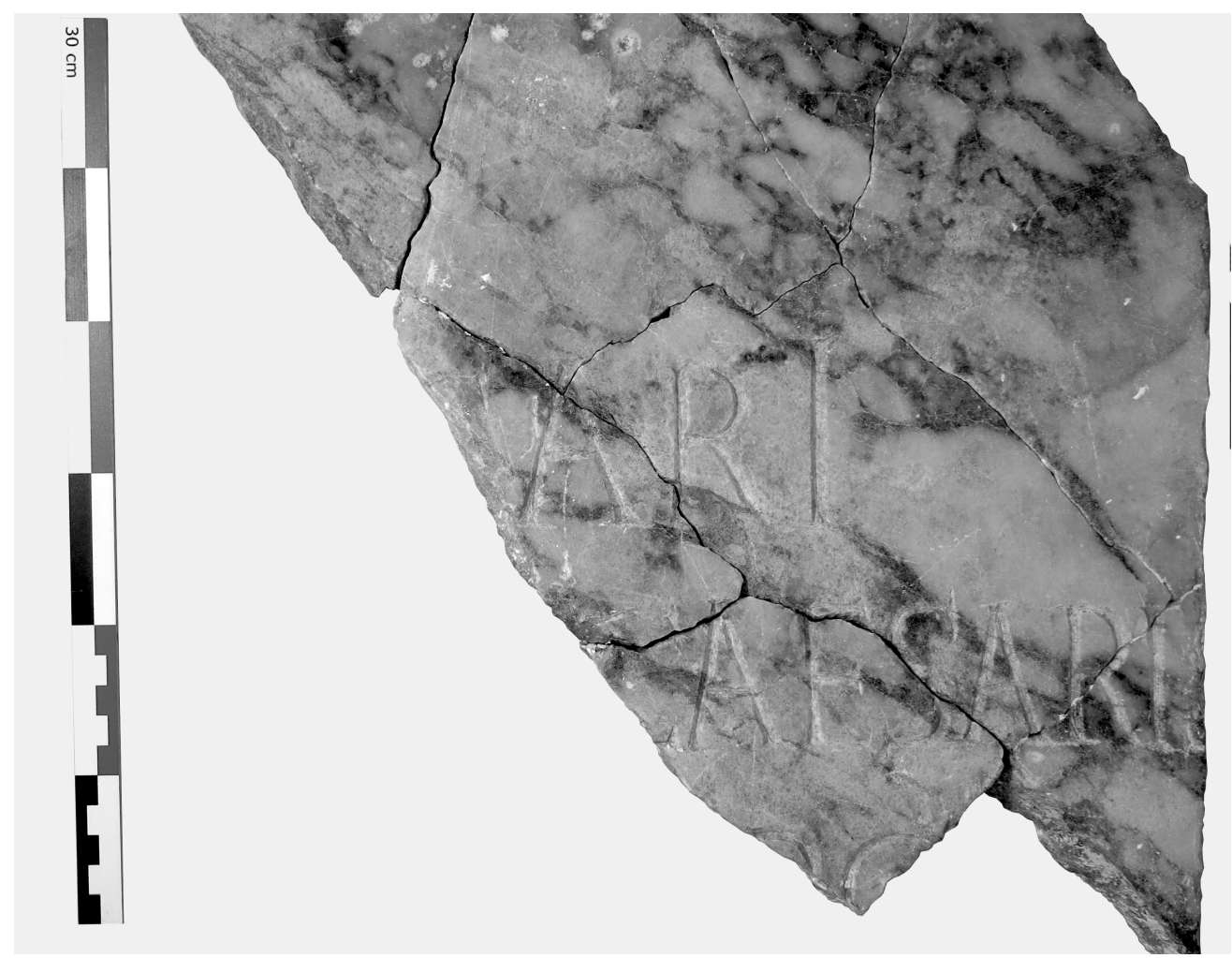

Figura 3. ILGN620.

un Valerius, aunque no tengamos más apoyo que los elementos formales comunes con ellas. La presencia de P. Memmius Regulus en el conjunto podría venir dada por el hecho de que este personaje hubiera sido uno de los fautores del ascenso social de los Valerii, que lo habrían recompensado con los honores ciudadanos y el patronato, además de incluirlo en un conjunto familiar de reconocimiento público junto con sus propias dedicatorias y estatuas, ya que no nos atrevemos a sugerir un parentesco efectivo.

Prestemos atención a continuación al contenido de dichas inscripciones, comenzando por las que nos parecen más antiguas; es decir, la grabadas sobre "pavonazzetto». La primera de ellas, ILGN $635=$ Gayraud $24=A E$ 1980, 615, reza como sigue (fig. 1): ${ }^{5}$

5. Sobre esta inscripción y su formulario, véase: Wierschowski, 1986: 287-294, esp. 288, donde da una nueva propuesta de lectura de dicha inscripción: Publice ex d(ecreto) d(ecurionum) / C(aio) Valerio, L(ucii) f(ilio), / Paeto, / primo [pilo, pra]ef(ecto) / fabrum, primo [inter s]uos / p[ont(ifici), fl]am[in]i / honor[is et virtutis causa] / Anni[a- - -], una restitución inteligente, pero que al no trabajar sobre los propios fragmentos del epígrafe resulta poco aproximada a la realidad de la pieza. Cf. PIR ${ }^{2}$ VIII/2, V 130: 73, donde J. Heinrichs propone que pudiera ser el padre del $P$. Valerius Maxumus que veremos a continuación. 


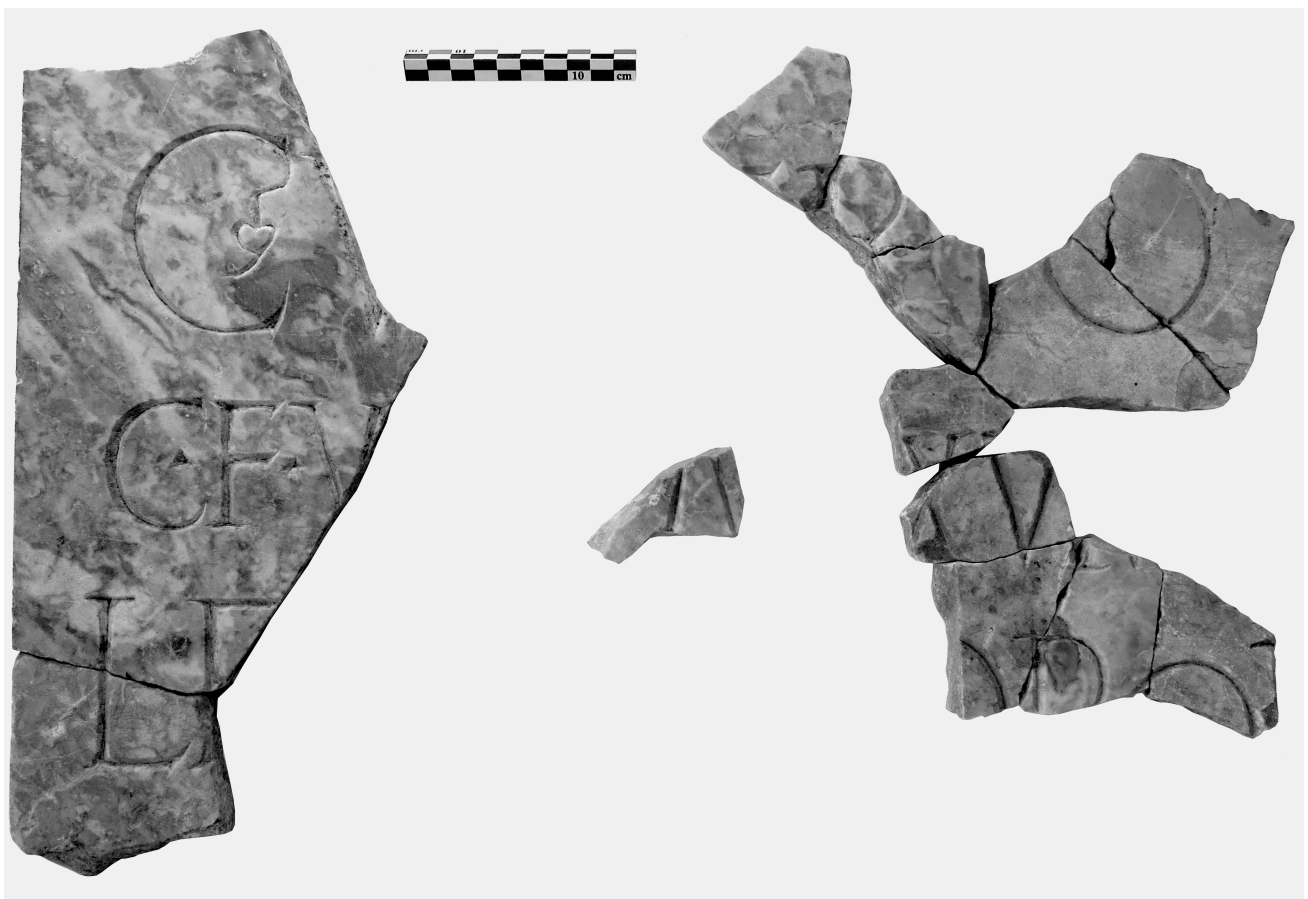

Figura 5. ILGN $636=$ Gayraud 22

dado que parece que podrían implicar otras cuestiones distintas de una simple sucesión de cargos en una fecha relativamente temprana. Es notable la reiteración de las constataciones de primo inter suos y primo in colonia sua, ${ }^{7}$ que tienen también su reflejo en el cursus anónimo que atribuimos a un Valerius que veremos a continuación. ${ }^{8}$

La segunda opistógrafa, Gayraud 5 b, con una dedicatoria a Calígula en la otra cara (fig. 3), ${ }^{9}$ lleva el texto siguiente (fig. 2):

7. La fórmula inter suos tiene solo paralelos en la Gallia Lugdunensis con la forma omnibus honoribus inter suos functo, CIL XIII 1695 y 2870. Con primus, como es nuestro caso, parece ser un semel dictum. Tampoco primus acompañado de in colonia sua es frecuente; hay ejemplos parecidos en África, así en Cirta , CIL VII $7041=$ 19423, primus in colonia y también en Icosium, CIL VIII 20853. Primus in colonia sua aparece en dos casos: en Thuburnica, CIL VIII 25742 y AE 1988, 1116 y también en esta misma ciudad, CIL VIII 14703, en la forma omnibus honoribus in colonia sua functis. En las Galliae podemos mencionar el caso de Arausio, CIL XII 1236, primus in colonia. La inscripción del famoso pantomimo Pylades de Ostia, CIL XIV 4624a, indica primo in coloniam.

8. Cf. nuestra nota 12 para este tipo de formulario.

9. E. Esperandieu no recoge este texto en ILGN (cf. nuestra nota 2), sino tan solo el de la inscripción imperial de la otra cara. El texto referido al emperador Gayo, Calígula, es el siguiente: [C(aio) Cae]sari / [Germanici] Caesaris / [filio Ti(berii) Caesaris Aug(usti) ne]pọ[ti] / - - - - - . Sobre esta inscripción cf., además de ILGN 620 y Gayraud,1980: 76, n. ${ }^{\circ}$ 5a; Hurlet, 1997: 598-599, n. ${ }^{\circ}$ 148; Rosso, 2000: 202-222, esp. 220, n. ${ }^{\circ}$ 15; Rosso, 2006: 406-407, n. ${ }^{\circ}$ 178. J. Gascou propone como alternativa posible la restitución en la primera línea de [Neroni Cae]sari, con lo cual la inscripción sería anterior a 29 dC, cuando este personaje fue declarado enemigo público por el senado. Cf. Gascou y Terrer 1996: 31-67, esp. 49-65 (J. Gascou); para este caso, p. 56, n. 17. 


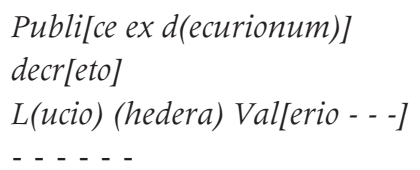

La serie realizada en "bardiglio" de Luni-Carrara está constituida, como ya hemos anunciado, por dos inscripciones seguramente atribuibles a los Valerii y una tercera anónima que nos parece también susceptible de asignación a los mismos.

Veamos a continuación la primera de ellas, ILGN 636 = Gayraud 22 ( fig.5): ${ }^{10}$

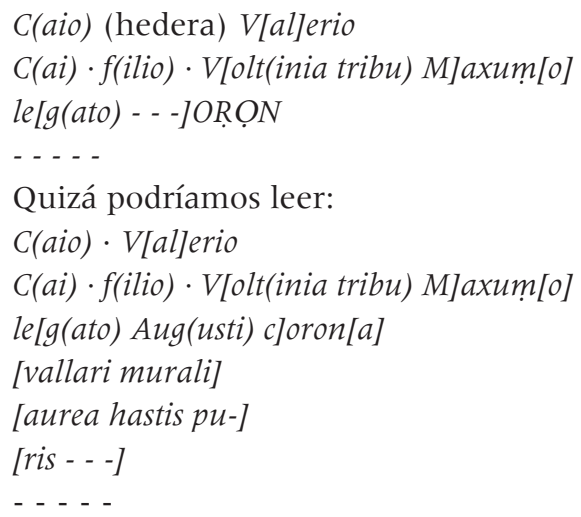

C. Valerius Maxumus es tal vez el único senador conocido seguro de Ruscino que habría nacido en la ciudad; ${ }^{11}$ en nuestra reconstrucción habría podido seguir una destacada carrera militar que podría haber conducido a su promoción. ${ }^{12}$

La segunda, $A E$ 1914, $27=I L G N 632=$ Gayraud $23=A E$ 1994, 1179 (fig. 6), aunque no indica el nomen, podemos atribuirla a un Valerius por el cognomen Maxumus y se dataría en 41-54 dC, según M. Gayraud, como veremos a continuación al intentar establecer un stemma de la familia:

$$
\begin{aligned}
& \text { [Publice ex d(ecurionum) d(ecreto)] } \\
& {[\text { C. Valerio M]axumo }} \\
& \text { tri[b(uno) } \cdot c] \text { hh (ortis) III } \cdot \text { praetoria [e] } \\
& \text { primo } \cdot \text { pilo } \cdot \text { bis }((\text { hedera }))
\end{aligned}
$$

10. Gayraud, $1980: 87$, n. ${ }^{\circ} 22=$ ILGN 636, con una lectura diferente del último renglón conservado siguiendo a Thiers, 1914: 184-194, esp. 192-193, donde restituye: le[g(ato) Aug(usti)] prop[r(aetore)] / [prov(inciae) - - -].

11. Burnand, 1982: 421; PIR ${ }^{2}$ VIII/2, V 130, Leipzig, 2015: 72-73 (J. Heinrichs); este personaje quizá fue hijo de C. Valerius Paetus ILGN $635=$ AE 1980, $615=$ AE 1987, 750, según se proponía también en REXV, Stuttgart 1955, S.v. "240) C.V[al]erius C. f. V[olt][Ma]xumus", col. 116.

12. Sobre las coronae y sus tipos, cf. Maxfield, 1981: 61-62. 


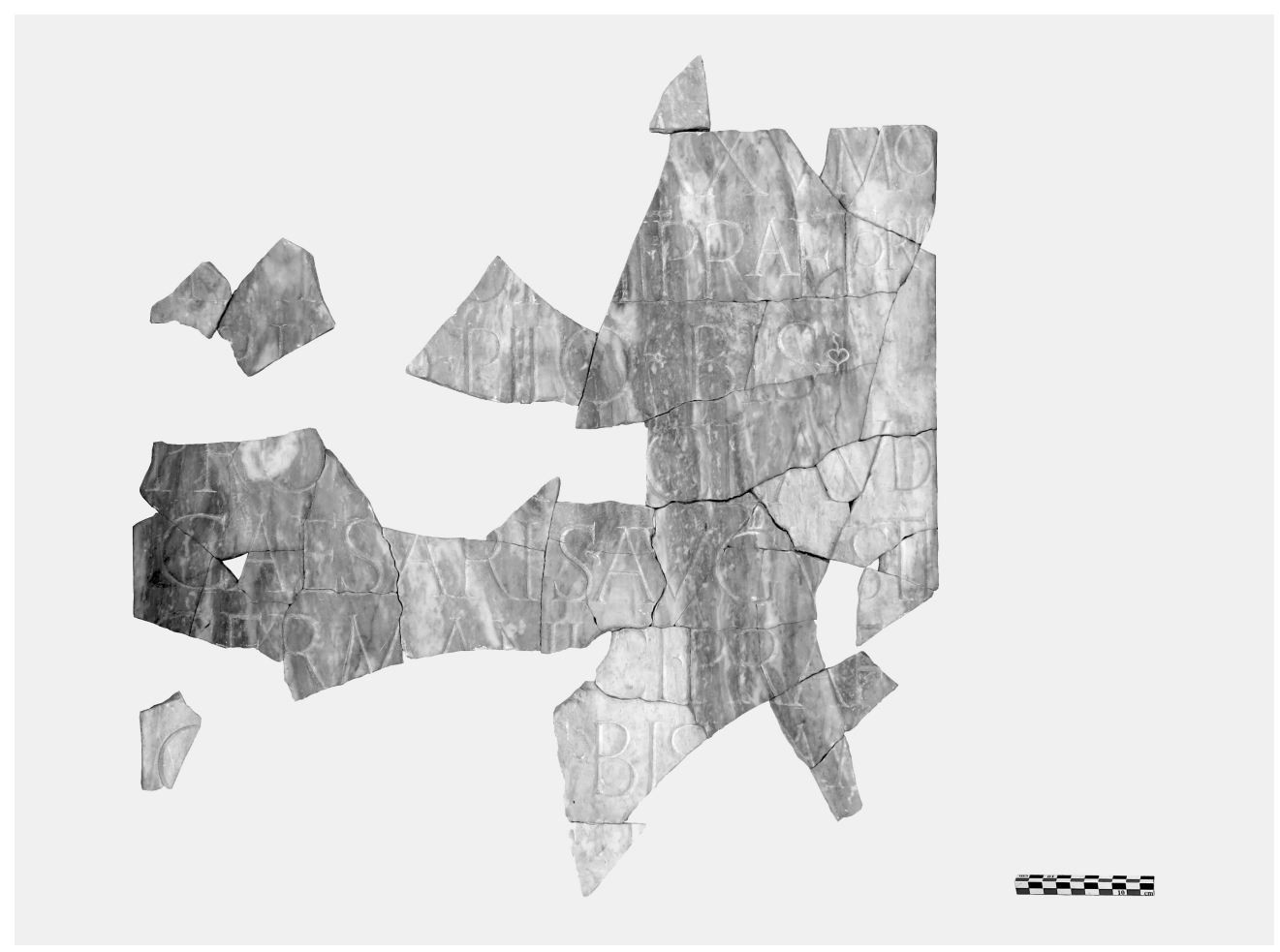

Figura 6. $A E 1914,27=I L G N 32=$ Gayraud $23=A E 1994,1179$.

proc[u]r(atori) $\cdot$ T[i(beri) $\cdot]$ Claudi
Caesaris $\cdot$ Augusti
Germanici $\cdot$ prae $[f($ ecto $)]$
C[aesari]s $\cdot$ bis f[l]am $($ ini)
[prov (inciae) bi]s $\cdot$ p[atrono]

El cursus de este personaje ha sido repetidamente estudiado. El problema fundamental continúa siendo su prefectura, que resulta indeterminada, aunque hayamos situado un con una $\mathrm{C}$ al inicio del renglón que la determina fragmento por lo cual praefectus Caesaris sería posible y menos probalemente praefectus classis, como quiso B. Dobson, quizá movido por los fragmentos que no parecen pertinentes de la inscripción siguiente. ${ }^{13}$

13. Pflaum, 1960: 28- 30, n. ${ }^{\circ} 12$, que da una transcripción en la que supone en r. 7-8: prae[fecto legion]s bis, unidad que en su opinión sería una de las legiones acantonadas en Egipto; reconoce, sin embargo, la ausencia de espacio para legionis en r. 8 y propone una división en dos líneas del término, que no resultaría necesaria a la vista de la pieza. Más recientemente, Dobson, 1978: 192, n. ${ }^{\circ}$ 58; Eck, 1994: 227-232, esp. 228, donde reconstruye: proc[u] r. T[i]. Claudi Caesaris August[i] Germanici prae[f. - - -]s. 
La tercera, anónima, solo puede ser atribuida a los Valerii por semejanzas formales, que incluso, como ya hemos visto, indujeron a atribuir fragmentos de la misma a la anterior, también incompleta.

Su texto, recogido parcialmente en $A E$ 1914, $28=I L G N 632=$ Gayraud $20=A E 1980$, 613 (fig. 7), viene integrado en nuestra lectura como sigue:

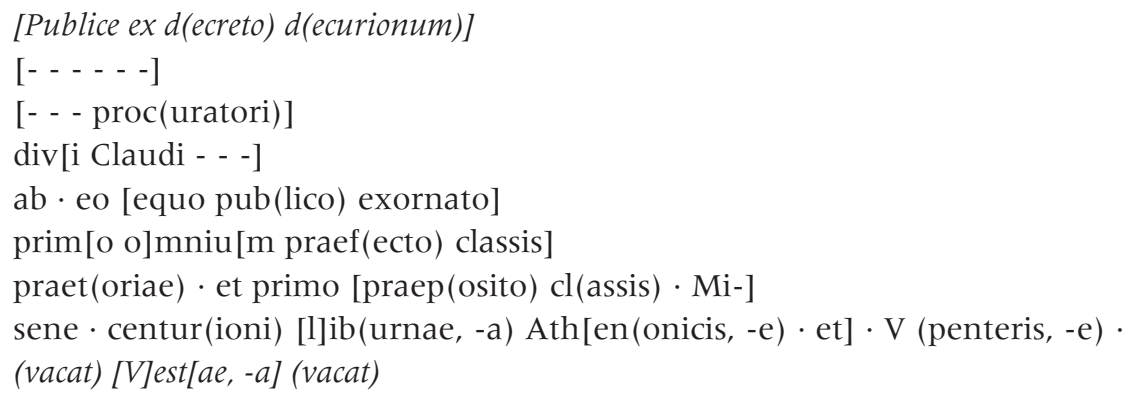

Como ya tuvimos ocasión de indicar, ${ }^{14}$ este documento parece tener una especial trascendencia para datar la constitución de la flota de Miseno, ya que permitiría en función de los datos internos fecharla en época claudiana, ya que el personaje anónimo fue seguramente praefectus de la classis praetoria de Miseno, primus ómnium, ${ }^{15}$ y recibió el equus publicus del emperador. ${ }^{16}$ La denominación praetoria para la classis de Miseno, en razón de su cronología, resulta extraña; la inscripción menciona, además, quizás al primer praefectus en esta condición, lo que nos llevaría, como hemos dicho, a pensar en las reformas navales de Claudio. ${ }^{17}$ Si se mantuvieran las fechas tradicionalmente aceptadas para la otorgación del título praetoria a la flota de Miseno, se debería replantear el momento final del conjunto forense de Ruscino más allá de la época flavia, lo que arqueológicamente no parece por el momento posible. Hay que decir también que hemos restituido los nombres de las naves a partir de ejemplos conocidos que pudieran adecuarse a los fragmentos conservados, sin que en principio podamos ofrecer al respecto seguridad alguna. ${ }^{18}$

Por otra parte, este epígrafe parece darnos una buena muestra de la promoción, en este caso "classiaria», de un personaje que muy bien pudiera ser uno de los Valerii de la segunda generación. Recordemos además que conocemos Valerii Paeti vinculados a las

14. En Mayer, 2016a: 142-143 y fig. 17, que incluye menos fragmentos que nuestra reconstrucción actual.

15. Sobre la fórmula relativamente frecuente utilizada, cf. Mrozek, 1971: 60-69.

16. Sobre la carrera de estos praefecti, cf. Kienast ,1966: 29-47.

17. Sobre esta classis Misenatium, cf. Reddé, 1986: 186-197, 490-492, 503-506 y, esp., 472, 511 y 515-518, donde destaca que el título de praetoria fue dado a las flotas principales por Vespasiano o Domiciano, y seguramente entre el año 71 y el $114 \mathrm{dC}$.

18. Cf. para un elenco de estos nombres Reddé, 1986: 665-672. Véase también el elenco puesto al día por Buonopane, 2017: 113-130, esp. 144 para Athenonice, y 120-121 y 124, para Vesta. 


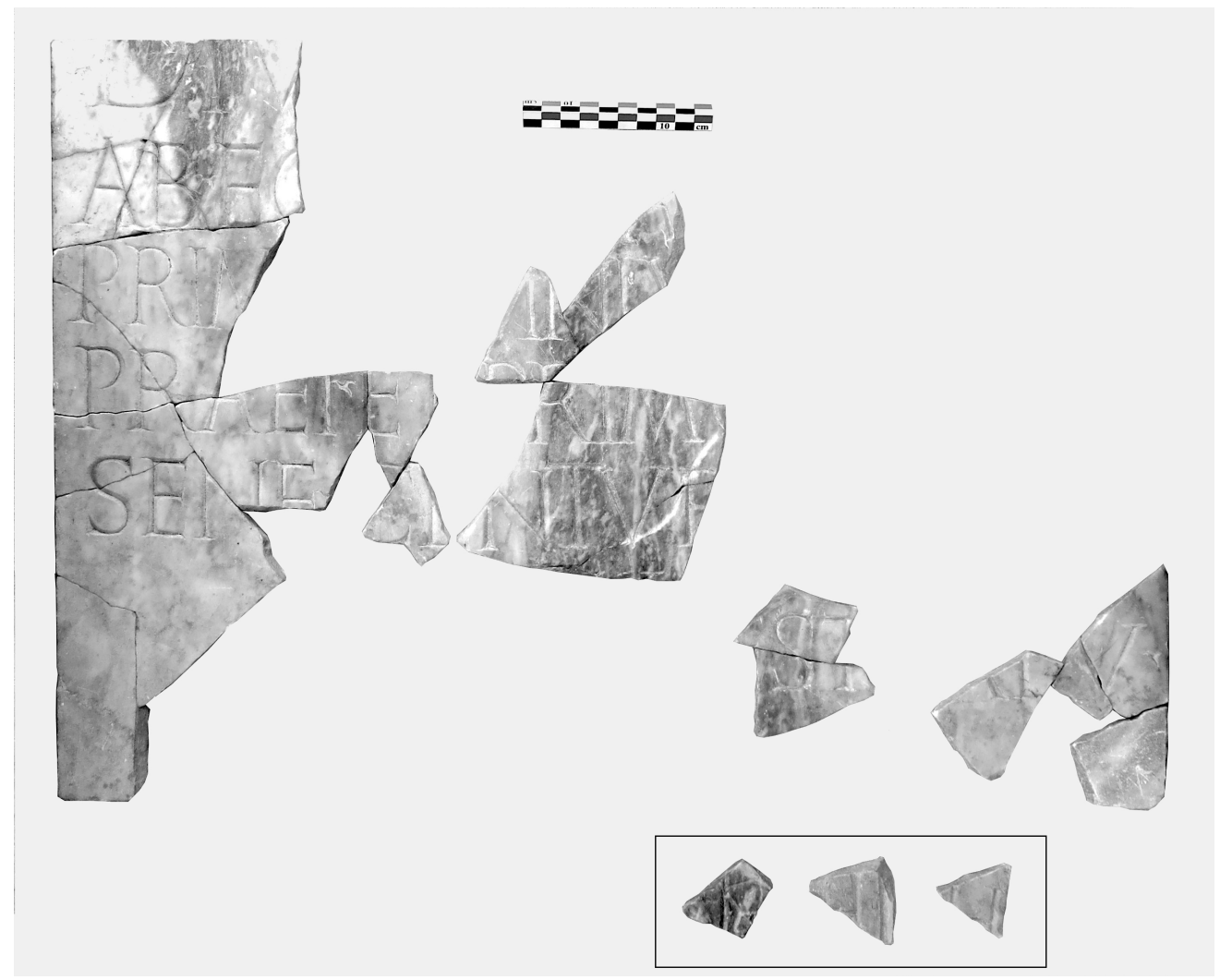

Figura 7. Inscripción cuyo texto es recogido parcialmente en $A E 1914,28=\operatorname{LLGN} 632=$ Gayraud $20=A E 1980,613$.

dos classes romanas, ${ }^{19}$ lo que quizá podría contribuir a reforzar cuanto decimos respecto a esta posible atribución.

Para poder evaluar correctamente esta posibilidad, deberemos centrarnos en suponer un posible stemma genealógico simple de los Valerii conocidos en Ruscino.

Dicho stemma podría partir de dos hermanos, en primera generación el C. Valerius Paetus y el L. Valerius de las inscripciones en "pavonazzetto", que tuvieron a su vez sendos hijos cuyos cursus recogerían las inscripciones de "bardiglio»: C. Valerius Maxumus en el primer caso y Maxumus, en el segundo, que tal vez pudo ser un (L. Valerius)

19. Conocemos Valerii Paeti praefectos de las flotas de Rávena y de Miseno: cf. PIR ${ }^{2}$ V, V. 156 p. $94-95$ (K. Wachtel, M. Heil). EI V.PAETI PR /CL PR.MIS, mencionado en un sello rectangular sobre un ladrillo de Blera, en la provincia de Viterbo (NSA 1882, p.166, n. $\left.{ }^{\circ} 14\right)$, podría estar relacionado con nuestro anónimo personaje, si no se trata del mismo. El Valerius Paetus conocido por dos diplomas militares, RMD 264 y 393, puede ser un descendiente, ya que dichos diplomas se datan en los años 142 y $145 \mathrm{dC}$, respectivamente. Para estos Valerii Paeti y su posible familia, cf. RE XV, "Valerius 282-284", cols. 173-174; también Reddé, 1986: 673-679, para un listado de los praefecti, esp. p. 674 para el Valerius Paetus, que lo fue en el año 145 dC, según se deprende de CIL XVI, 9. 
Maxumus, con lo que ambos primos llevarían el mismo cognomen de tradición seguramente familiar.

El personaje anónimo de la tercera inscripción grabada en "bardiglio», si supusiéramos que fue otro Valerius, podría haber sido hermano de uno de los anteriores, aunque sería por su carrera posiblemente de la rama de la familia que debió de permanecer en el ordo equester, ya que $C$. Valerius Maxumus alcanza el ordo senatorius a partir de un origen en el ordo equester, al que pertenece sin duda ya C. Valerius Paetus. No podemos precisar el nivel alcanzado por el L. Valerius de la inscripción opistógrafa, dado lo incompleto de su inscripción, aunque, visto el caso de Paetus, quizá superó la condición de notable local para llegar a la de caballero; el hecho de que se aprovechara una inscripción imperial de Calígula para rendirle honores podría apuntar también a un alto nivel social del personaje.

Como tuvimos ya ocasión de señalar, ${ }^{20}$ existen también otras posibilidades a partir del papel preponderante de los Valerii desde época augustea en Ruscino. Sir Ronald Syme ${ }^{21}$ por su parte sugirió que uno de los protegidos por el efímero emperador Galba, Valerius Marinus, que Tácito (Hist. 2, 71, 2) cita como designado para el consulado por el emperador, pudiera ser de la Galia Narbonense; si esto tuviera visos de certeza, deberíamos quizás abrirnos a la posibilidad de que se tratara también de un miembro de la familia de los Valerii de Ruscino, ya en el ordo senatorius.

Sobre el origen de esta familia tal vez pueda suponerse, como hemos propuesto en un trabajo anterior, ${ }^{22}$ que estuviera vinculado a la clientela narbonense de $C$. Valerius Flaccus, que fue procónsul de la Gallia Transalpina entre 85 y 81 aC. ${ }^{23}$ El momento de esta entrada en una clientela romana podría abonar su promoción posterior en razón de su fecha temprana. A las órdenes del gobernador estuvo como tribunus militum, en torno a $82 \mathrm{aC}$, su sobrino L. Valerius Flaccus, que sería pretor en el año 63 aC. ${ }^{24}$ Los praenomina de los Valerii de Ruscino coinciden con los de ambos personajes, lo que quizá sería un indicio significativo.

Por último, no sabemos hasta qué punto los Valerii vinculados a la flota pudieron estar relacionados también con la destrucción de Ruscino, ya que no sabemos qué partido debieron tomar, y si tuvieron algo que ver con la guerra civil que causó y siguió a la muerte de Nerón en la que intervinieron activamente en la Narbonensis las naves de guerra, ${ }^{25}$ un elemento más a añadir al expediente del final de la ciudad del que hemos tratado en otras ocasiones.

20. Mayer, 2016a: 143-144.

21. Syme, 1982: 460-483, esp. 477.

22. Mayer (e.p.), con una discusión más amplia.

23. Sería el cónsul del año 93 aC. Cf. MRR II, 14, para el consulado y 58-59, 61 y 70, para el gobierno provincial; además, MRR III, 211.

24. MRR II, 629 y MRR III, 212, para su carrera.

25. Kienast,1966: 61-65, esp. 63-64; Mayer, 2016c: 234-236. 


\section{Bibliografía}

BUONOPANE, A., 2017, Le navi delle flotte di Ravenna e Miseno e i loro nomi, en L. CHIOFFI, M. KAJAVA y S. ÖRMÄ (eds.), Il Mediterraneo e la storia II. Naviganti, popoli e culture ad Ischia e altri luoghi della costa tirrenica. Atti del convegno internazionale, Sant'Angelo di Ischia 9-11 ottobre 2015, Roma (Acta Instituti Romani Finlandiae, 45), $113-130$.

BURNAND, Y., 1982, Senatores Romani ex provinciis Galliarum orti, en Epigrafia e ordine senatorio, vol. II, Roma (Tituli 5), 387-437.

DOBSON, B., 1978, Die Primipilares, Colonia.

ECK, W., 1994, Prosopographica, ZPE 101, 227-232.

GASCOU, J., 1997, Magistratures et sacerdoces municipaux dans les cités de Gaule Narbonnaise, en M. CHRISTOL y O. MASSON (eds.), Actes $d u$ $X^{e}$ Congrès international d'épigraphie grecque et latine, Nîmes 4-9 octobre 1992, París, 75-140.

GASCOU, J. y TERRER, D., 1996, La présence de Tibère en Narbonnaise: les portraits et les inscriptions, RAN 29, 31-67.

GAYRAUD, M., 1980, Les inscriptions de Ruscino, en G. BARRUOL (ed.), Ruscino. Château-Roussillon, Perpignan (Pyrénées-Orientales), 1. Etat des travaux et recherches en 1975. Actes du Colloque archéologique organisé par la Direction des antiquités historiques du Languedoc-Roussillon, Perpignan 1975, Revue archéologique de Narbonnaise, supplément 7. París, 67-97.

HURLET, F., 1997, Les collègues du prince sous Auguste et Tibère. De la légalité républicaine à la légitimité dynastique, Collection de l'École Française de Rome 227, Roma.

$I L G N=$ E. ESPERANDIEU, Inscriptions latines de Gaule (Narbonnaise), París, 1929.

KIENAST, D., 1966, Untersuchungen zu den Kriegsflotten der römischen Kaiserzeit, Bonn (Antiquitas, Reihe 1, 13).

MAYER, M., 2016a, El espectáculo epigráfico del foro de Ruscino: un horizonte efímero, en A. DONATI (ed.), L'iscrizione esposta. Atti del Convegno Borghesi 2015, Faenza (Epigrafia e antichità 37), 121-145.

MAYER, M., 2016b, Consideraciones sobre el fórum de Ruscino y su horizonte epigráfico, en G. BARATTA (ed.), Studi su Ruscino, Barcelona (Sylloge Epigraphica Barcininensis. Annexos II), 19-44.

MAYER, M., 2016c, Los honores dinásticos de los Flavios y el precedente de Galba: la significación política de dos inscripciones de Tarraco, CIL II ${ }^{2}$ 881 y 894, en E. REDONDO-MOYANO y M.J. GARCÍA SOLER (eds.), Nuevas interpretaciones del Mundo Antiguo. Papers in Honor of Professor José Luis Melena on the Occasion of his Retirement, VitoriaGasteiz 2016 (Anejos de Veleia. Series Minor, 339), 233-243.

MAYER, M. (e.p.), Ruscino y Publius Memmius Regulus.

MAXFIELD, V.A., 1981, The Military Decorations of the Roman Army, Berkeley, Los Ángeles.

MROZEK, S., 1971, Primus omnium sur les inscriptions des municipes italiens, Epigraphica 33, 60-69.

MRR II = T.R.S. BROUGHTON, The Magistrates of the Roman Republic, vol. II, Cleveland, Ohio 1968, reimpr. con adiciones de la ed. de 1952 (Phil. Monographs of the American Philological Association XV).

MRR III = T.R.S. Broughton, The Magistrates of the Roman Republic, vol. III supplement, Atlanta, Georgia, 1986 (Phil. Monographs of the American Philological Association, XV, 3).

PFLAUM, H.G., 1960, Les carrières procuratoriennes équestres sous le Haut-Empire romain, vol. I, París 1960.

PFLAUM, H.G., 1978, Les Fastes de la province de Narbonnaise $\left(\mathrm{XXX}^{\mathrm{e}}\right.$ supplément à "Gallia»), París.

REDDÉ, M., 1986, Mare nostrum. Les infrastructures, le dispositif et l'histoire de la marine militaire sous l'Empire romain, Roma (BEFAR, 260). 
ROSSO, E., 2000, La série de dédicaces julioclaudiennes de Ruscino, Château-Roussillon (Perpignan, Pyrénées-Orientales), RAN 33, 1, 202-222.

ROSSO, E., 2006, L'image de l'empereur en Gaule romaine: portraits et inscriptions, París, 2006 (Archéologie et histoire de l'art, 20).

SYME, R., 1982, Partisans of Galba, Historia, XXI, 460-483.
THIERS, F.P., 1914, Rapport sur les fouilles de Castel-Roussillon (Pyrénées-Orientales) en 1913, Bulletin Archéologique du Comité des Travaux Historiques et Scientifiques, 184-194.

WIERSCHOWSKI, L., 1986, AE 1980, 615 und das erste Auftreten der Formel 'Omnibus Honoribus in Colonia Sua Functus' in den westlichen Provinzen, ZPE, LXIV, 287-294. 